\title{
Natural Convection in a Horizontal Porous Annulus under Quasi-periodic Gravitational Modulation
}

\author{
Jabrane Belabid $^{1, \mathrm{a}}$, Karam Allali ${ }^{1, \mathrm{~b}}$, and Mohamed Belhaq ${ }^{2, \mathrm{c}}$ \\ 1 University Hassan II-Casablanca, FSTM, Department of Mathematics, PO Box Mohammadia, Morocco \\ 2 University Hassan II-Casablanca, FS, Department of Physics, Casablanca, Morocco
}

\begin{abstract}
The effect of quasi-periodic gravitational modulation on the natural convection in a horizontal porous annulus is studied in this paper. The model we consider includes the heat and the hydrodynamics equations under Darcy law. The derived model with the stream function is solved numerically using the alternate direction implicit method. Results show that the convective instability and the flow regime are influenced by frequencies ratio of the quasi-periodic gravitational modulation.
\end{abstract}

\section{Introduction}

Natural convection and heat transfer in porous media has been motivated by a wide range of engineering application such as cooling of electronic equipments, aircraft cabin insulation, geothermal systems, underground transmission lines, storage of nuclear waste materials, solidification of casting to name just few (see for instance [1-3], and references therein).

The pioneer work of Caltagirone [4] studied the free convection flows in a porous horizontal annulus heated from the inner cylinder. The author observed different flow regimes when the Rayleigh number exceeds some critical value. Later, many authors tackled the same problem of natural convection in porous annulus. For example, Rao et al. [5] studied the phenomena of two-dimensional bifurcation using the Galerkin method to solve the governing equations. The critical Rayleigh numbers determined numerically are in a very good agreement with the experimental results and with those of Caltagirone [4]. Himasekhar and Bau [6] investigated the problem using the regular perturbation expansion for different radii ratio.

Recently Belabid and Allali [7] studied the effect of periodic gravitational modulation on convective instability in horizontal annulus. It was shown that the convective instability depends on the modulation amplitude and frequency. Although, the influence of periodic gravitational modulation on convective instability is widely studied, only few works considered the effect of quasi-periodic (QP) gravitational modulation on the convective instability. For instance, it was shown than the frequencies ratio has a stabilizing or destabilizing effect on the onset of convection for the problems dealing with reaction front propagation $[8,9]$ and for the problems of Hele-shaw cell $[10,11]$. Motivated by the recent work by Belabid and Allali [7], the main objective of the present paper is to examine the effect of the QP gravitational modulation on the convective instability in a porous horizontal annulus. To this end, we assume that

\footnotetext{
a e-mail: belabide@gmail.com

b e-mail: allali@hotmail.com

c e-mail: belhaq@yahoo.fr
}

the annulus is subject to QP gravitational modulation with two incommensurate frequencies.

The rest of the work is organized as follows. The problem description is given in the next section. The numerical method is illustrated in Section 3, and in Section 4 numerical results are given. Section 5 concludes the work.

\section{Problem description and mathematical formulation}

We consider a porous layer bounded by two horizontal concentric cylinders saturated with a Newtonian fluid as shown in Fig. 1. Both inner and outer cylinders are kept at uniform, constant and different temperature $T_{i}$ and $T_{o}$ respectively with $T_{i}>T_{o}$. It is taken into account that the

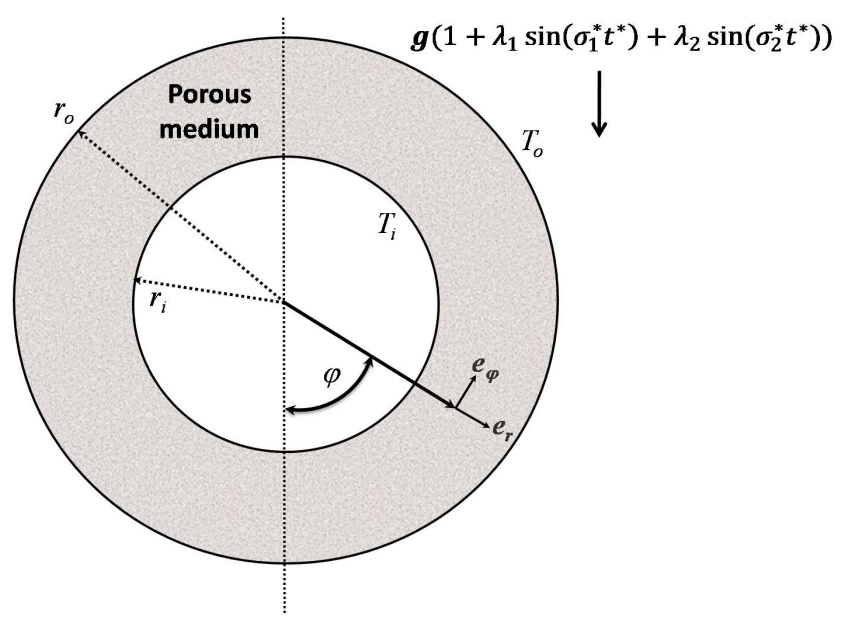

Fig. 1. Sketch of the problem

flow is two-dimensional, steady and laminar. The porous medium is considered to be homogenous, isotropic and subjected to a QP gravitational modulation with two incommensurate frequencies. Under the Boussinesq approximation, the basic steady conservation of thermal energy, 
momentum and mass equations in polar coordinates system can be written as:

$$
\begin{gathered}
\frac{\partial T^{*}}{\partial t^{*}}+\left(\mathbf{V}^{*} \cdot \nabla\right) T^{*}=\alpha \nabla T^{*} \\
\mathbf{V}^{*}=-\frac{K}{\mu}\left(\nabla P^{*}-\rho \mathbf{g}\left(1+\lambda_{1} \sin \left(\sigma_{1}^{*} t^{*}\right)+\lambda_{2} \sin \left(\sigma_{2}^{*} t^{*}\right)\right)\right) \\
\nabla \cdot \mathbf{V}^{*}=0
\end{gathered}
$$

with the following boundary conditions:

On the inner cylinder surface: $u^{*}=0, T^{*}=T_{i}$.

On the outer cylinder surface: $v^{*}=0, T^{*}=T_{o}$,

where $T^{*}$ is the temperature, $V^{*}$ is the velocity, $P^{*}$ is the pressure, $\alpha$ is the diffusion, $K$ the permeability, $\mu$ the viscosity, $\rho$ the density, $\lambda_{i}$ and $\sigma_{i}^{*}$ stand for the amplitudes and the frequencies of the QP modulation, respectively $(i=1,2)$.

To obtain the dimensionless model, we introduce the following transformation formula:

$r=\frac{r^{*}}{r_{i}}, \mathbf{V}=\frac{\mathbf{V}^{*}}{\alpha} r_{i}, T=\frac{T^{*}-T_{o}}{T_{i}-T_{o}}, t=\frac{t^{*}}{r_{i}^{2}} \alpha, P=\frac{P^{*} K}{\alpha \mu}$,

$\sigma_{1}=\frac{\sigma_{1}^{*} r_{i}^{2}}{\alpha}$ and $\sigma_{2}=\frac{\sigma_{2}^{*} r_{i}^{2}}{\alpha}$. Therefore, the dimensionless governing equations can be rewritten as:

$$
\begin{gathered}
\frac{\partial T}{\partial t}+(\mathbf{V} \cdot \nabla) T=\nabla T \\
\mathbf{V}=-\nabla P-\operatorname{RaT} \boldsymbol{k}\left(1+\lambda_{1} \sin \left(\sigma_{1} t\right)+\lambda_{2} \sin \left(\sigma_{2} t\right)\right) \\
\nabla \cdot \mathbf{V}=0
\end{gathered}
$$

where $R a$ is the Rayleigh number given by $R a=\frac{g \beta K \Delta T r_{i}}{\alpha v}$; and $\sigma_{2}=\eta \sigma_{1}$. The dimensionless boundary conditions are as follows:

On the inner cylinder surface: $u=0, T=1$.

On the outer cylinder surface: $v=0, T=0$.

Due to the physical symmetry of the problem the following boundary conditions are added:

$\varphi=0, \pi: v=0, \frac{\partial T}{\partial \varphi}=0$

The governing equations for the laminar vibrated natural convection in terms of stream function-temperature formulation can be expressed as follows:

$$
\begin{gathered}
\nabla^{2} \psi=-R a\left(\sin \varphi \frac{\partial T}{\partial r}+\frac{\cos \varphi}{r} \frac{\partial T}{\partial \varphi}\right)\left(1+\lambda_{1} \sin \left(\sigma_{1} t\right)+\lambda_{2} \sin \left(\sigma_{2} t\right)\right) \\
\frac{\partial T}{\partial t}+\frac{1}{r} \frac{\partial \psi}{\partial \varphi} \frac{\partial T}{\partial r}-\frac{1}{r} \frac{\partial \psi}{\partial r} \frac{\partial T}{\partial \varphi}=\nabla^{2} T
\end{gathered}
$$

and the corresponding boundary conditions take the following form:

$r=1: T=1$ and $\frac{\partial \psi}{\partial \varphi}=0 ;$

$r=R: T=0$ and $\frac{\partial \psi}{\partial \varphi}=0 ;$

$\varphi=0, \pi: \frac{\partial T}{\partial \varphi}=\frac{\partial \psi}{\partial r}=0$.

The local Nusselt number along the inner and outer cylinders are estimated as the ratio of convective to conductive heat transfer:

$$
N u_{i}(r=1, \varphi)=-\left.\ln R \frac{\partial T}{\partial r}\right|_{r=1}
$$

$$
N u_{o}(r=R, \varphi)=-\left.R \ln R \frac{\partial T}{\partial r}\right|_{r=R}
$$

The average Nusselt numbers evaluated at the inner and outer cylinders can be written as:

$$
\begin{gathered}
\overline{N u}=\frac{1}{\pi} \int_{0}^{\pi} N u_{i} d \varphi=-\left.\frac{1}{\pi} \ln R \int_{0}^{\pi} \frac{\partial T}{\partial r}\right|_{r=1} d \varphi \\
\overline{N u}=\frac{1}{\pi} \int_{0}^{\pi} N u_{o} d \varphi=-\left.\frac{1}{\pi} R \ln R \int_{0}^{\pi} \frac{\partial T}{\partial r}\right|_{r=R} d \varphi
\end{gathered}
$$

For a sufficient refined mesh, both expressions of the average Nusselt number converge to the same value.

\section{Numerical methods and code validation}

The alternate direction implicit finite difference method is used to solve the mathematical problem along with boundary conditions. The Thomas algorithm in conjunction with iterations is used to solve the resulting matrix systems. The convergence of our in-house numerical code has been assessed by a mesh testing procedure. In fact, numerical tests, using various mesh grids, were examined for the case without any modulation i.e. $\lambda_{i}=0, i=1,2$; in order to determine the best compromise between the grid independent solutions and the calculation time. The iteration process is terminated when the following criterion is satisfied in each node of the grid:

$$
\max \left|\frac{\xi_{i, j}^{n+1}-\xi_{i, j}^{n}}{\xi_{i, j}^{n}}\right|<10^{-8}
$$

\begin{tabular}{lccc}
\hline & Grid size & $R a=50$ & $R a=100$ \\
\hline Caltagirone [4] & $49 \times 49$ & 1.328 & 1.829 \\
\hline Facas and Farouk [14] & $25 \times 25$ & 1.362 & 1.902 \\
\hline Rao et al. [6] & $10 \times 10$ & 1.341 & 1.861 \\
\hline Facas [15] & $50 \times 50$ & 1.342 & 1.835 \\
\hline Charrier-Mojtabi [16] & $30 \times 95$ & 1.344 & 1.867 \\
\hline Mota et al. [17] & $161 \times 101$ & 1.338 & 1.861 \\
\hline Alfahaid et al. [12] & $10 \times 18$ & 1.317 & 1.865 \\
\hline Alloui and Vasseur [13] & $100 \times 240$ & 1.343 & 1.868 \\
\hline This study & $49 \times 49$ & 1.343 & 1.851 \\
\hline
\end{tabular}

Table 1. Nusselt number for $R=2$. Comparison with literature

where $\xi$ refers to $T$ or $\psi$, the subscripts $i$ and $j$ indices denote grid location in the $(r, \varphi)$ plane and $n$ designates the iteration number. The present code was validated by comparing the obtained result for $R a=50$ and $R a=100$ with the already published data (see Table 1). Figure 2 shows that we can choose the mesh grid $71 \times 71$ for our numerical simulations which ensures the mesh grid independence.

\section{Results and discussions}

The numerical simulation will be performed for the fixed amplitude $\lambda_{1}=\lambda_{2}=0.1$, the frequency $\sigma_{1}=10$ and the radius $R=2$. The frequencies ratio $\eta$ and the Rayleigh number will be varied adequately in order to check the QP 


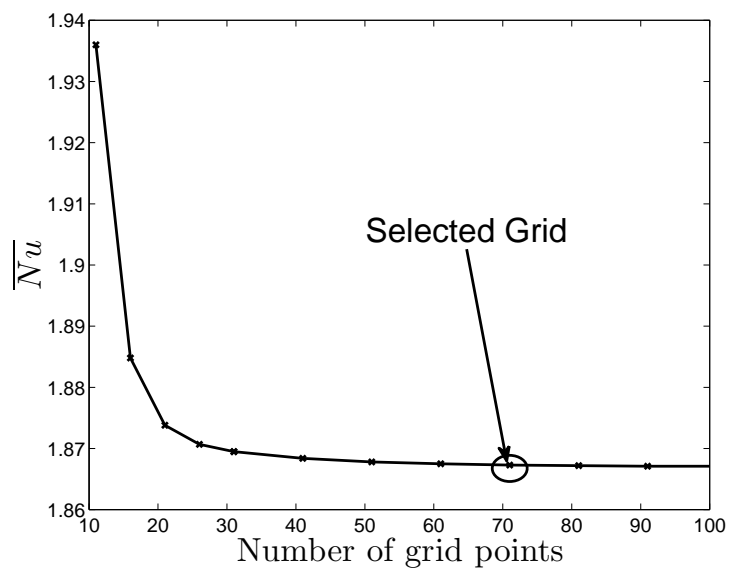

Fig. 2. Mesh effect on $\overline{N u}$ for $\lambda_{1}=\lambda_{2}=0$ and $R a=100$

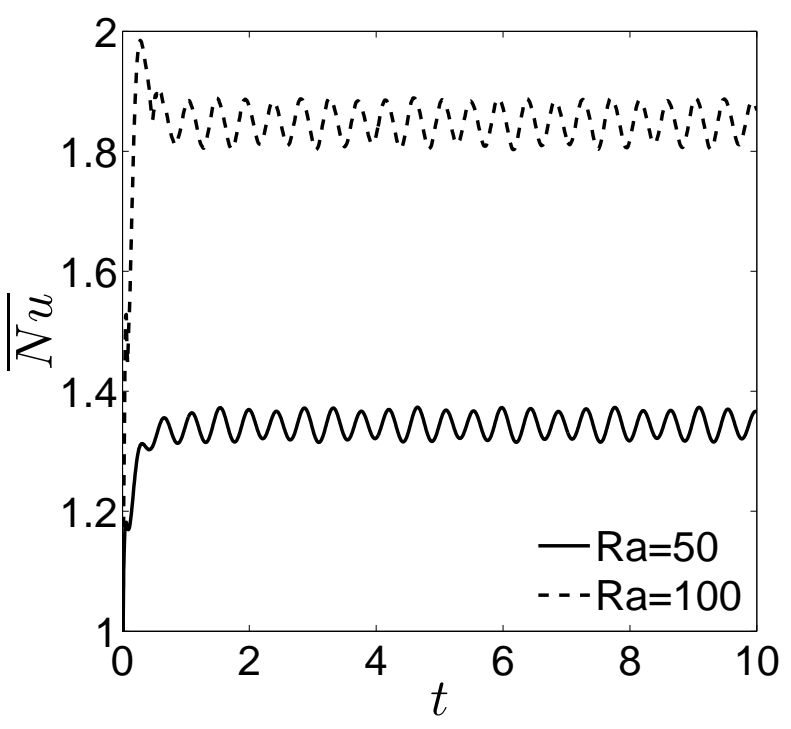

Fig. 3. Nusselt number as function of time for $\eta=\sqrt{2}$

modulation on the onset of convection. Figure 3 shows the Nusselt number as function of time for different Rayleigh number. First, we clearly observe a small modulation in the behaviour of the Nusselt number. On the other hand, increasing Rayleigh number leads to an increase of the heat transfer. The comparison between the case of periodic and QP gravitational modulation is illustrated in Fig. 4. Indeed, when the two frequencies ratio is zero we find exactly the results in [7]. By increasing the value of $\eta$ the heat transfer has an alternate behaviour around a mean value which means that a destabilizing effect of the QP gravitational modulation is observed. Finally, in Fig. 5, we observe a stabilizing effect for small frequencies ratio and a destabilizing effect for high ones since transition from unicellular to bicellular flow regime occurs by increasing the frequencies ratio.

\section{Conclusion}

In this paper, we have studied the effect of the QP gravitational modulation on convective instability in horizontal porous annulus. The gravitational modulation is assumed
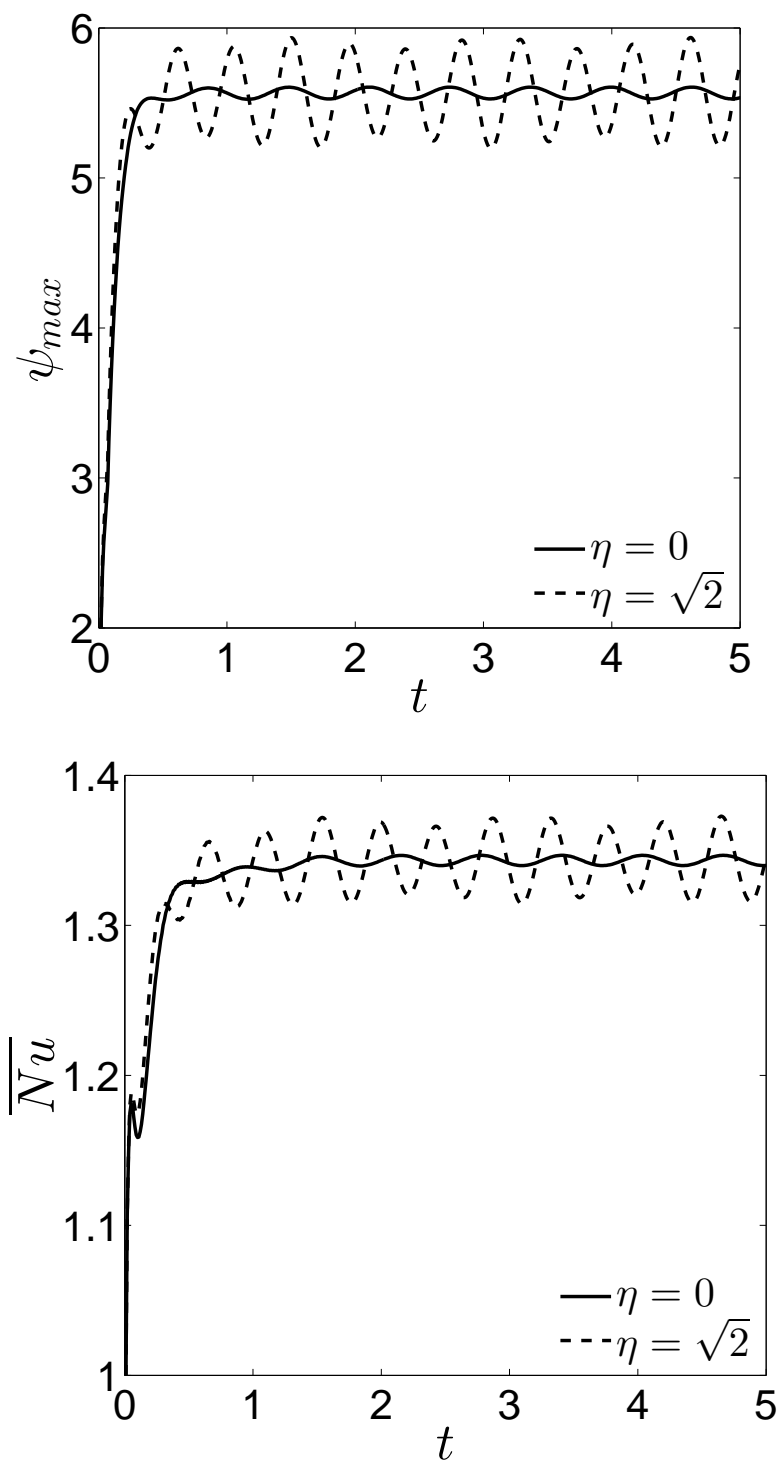

Fig. 4. Nusselt number and $\psi_{\max }$ as function of time for $R a=50$

to be with two incommensurate frequencies. The considered model consists of heat equation coupled with the hydrodynamics under Darcy law. The reduced model with the stream function formulation was solved using the alternate direction implicite method. It was shown that the convective instability depends on the frequencies ratio. More precisely, it was observed that the QP gravitational modulation has a destabilizing effect induced by an alternate behaviour of the Nusselt number.

\section{References}

1. P. Vadasz, Emerging topics in heat and mass transfer in porous media, Springer-Verlag, New York, 2008.

2. D.A. Nield, A. Bejan, Convection in porous media, $4^{\text {th }}$ ed., Springer-Verlag, New York, 2013.

3. K. Vafai, Handbook of porous media, $3^{\text {rd }}$ ed., Taylor \& Francis, New York 2015.

4. J.P. Caltagirone, Instabilities in a porous medium bounded by two concentric horizontal cylinders, Journal of Fluid Mechanics 76 (1976) 337-362. 
5. Y.F. Rao, K. Fukuda, S. Hasegawa, Steady and transient analysis of natural convection in a horizontal porous annulus with the Galerkin method, Journal of Heat Transfer 109 (1987) 919-927.

6. K. Himasekhar, H.H. Bau, Two-dimensional bifurcation phenomena in thermal convection in horizontal, concentric annuli containing saturated porous media, Journal of Fluid Mechanics 187 (1988) 267-300.

7. J. Belabid, K. Allali, Influence of gravitational modulation on natural convection in a horizontal porous annulus, Journal of Heat Transfer 139 (2017) 022502.

8. K. Allali, M. Belhaq, K. El Karouni, Influence of quasi-periodic gravitational modulation on convective instability of reaction fronts in porous media, Communications in Nonlinear Science and Numerical Simulation, 17 (2012) 1588-1596.

9. K. Allali, S. Assiyad, M. Belhaq, Convection of polymerization front with solid product under quasiperiodic gravitational modulation, Nonlinear Dynamics and Systems Theory, 14 (2014) 323-334.

10. T. Boulal, S. Aniss, M. Belhaq, R. Rand, Effect of quasiperiodic gravitational modulation on the stability of a heated fluid layer, Physical Review E, 76 (2007) 056320 .

11. T. Boulal, S. Aniss, M. Belhaq, A. Azouani, Effect of quasi-periodic gravitational modulation on the convective instability in Hele-Shaw cell, International Journal of Non-Linear Mechanics 43 (2008) 852-857.

12. A.F. Alfahaid, R.Y. Sakr, M.I. Ahmed, Natural convection heat transfer in concentric horizontal annuli containing saturated porous media, IIUM Engineering Journal 6 (2005) 41-54.

13. Z. Alloui, P. Vasseur, Natural convection in a horizontal annular porous cavity saturated by a binary mixture, Computational Thermal Sciences 3 (2011) 407 417.

14. G.N. Facas, B. Farouk, Transient and Steady State Natural Convection in a Porous Medium between Two Concentric Cylinders, Journal of Heat Transfer 105 (1983) 660-663.

15. G.N. Facas, Natural Convection From a Buried Pipe with External Baffles, Numerical Heat Transfer, Part A 27 (1995) 595-609.

16. M.C. Charrier-Mojtabi, Numerical simulation of twothree dimensional free convection flows in a horizontal porous annulus using a pressure and temperature formulation, International Journal of Heat and Mass Transfer 40 (1997) 1521-1533.

17. J.P.B. Mota, I.A.A.C. Esteves, C.A.M. Portugal, J.M.S.S. Esperana, E. Saatdjian, Natural convection heat transfer in horizontal eccentric elliptic annuli containing saturated porous media, International Journal of Heat and Mass Transfer 36 (2000) 4367-4379.

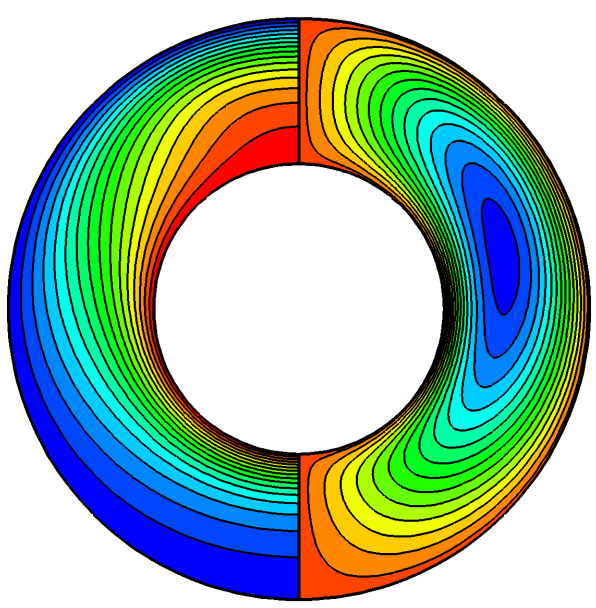

(a): $\eta=\sqrt{2}$

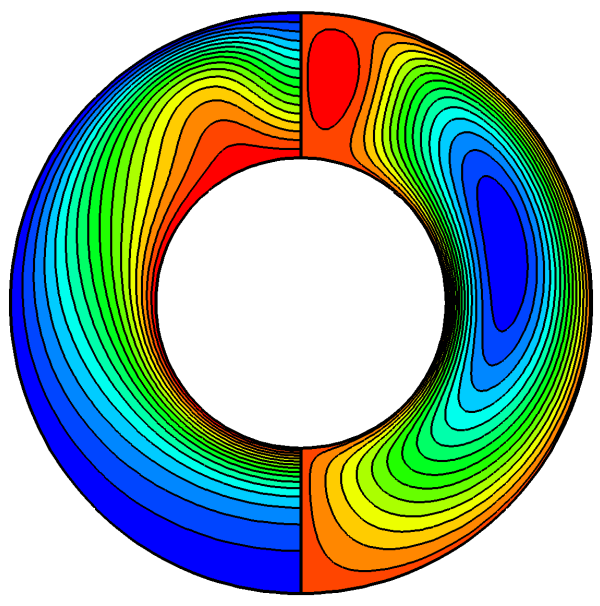

(b): $\eta=\sqrt{3}$

Fig. 5. Effect of $\eta$ on the flow structure for $R a=63.77$ 